\title{
Adenomyosis in Pakistani women: four year experience at the Aga Khan University Medical Centre, Karachi
}

\author{
H Shaikh, K S Khan
}

\begin{abstract}
As part of a quality assurance programme at the Aga Khan University Medical Centre, Karachi, Pakistan, all hysterectomy specimens were reviewed from January 1986 to December 1989. Adenomyosis was found in 237 of the 419 $(56.5 \%)$ specimens studied. Of these 237 patients, $232(97.9 \%)$ were parous and 196 $(82 \cdot 8 \%)$ were in the fourth and fifth decades of life. This high prevalence in parous women aged 40-59 years was significant. Fibroids, cervicitis, and endometrial hyperplasia were the most common associated diagnoses. Of all the associations studied, only endometrial hyperplasia was significantly more prevalent in the group with adenomyosis. Adenomyosis was stated as an indication for surgery in 69 patients and was confirmed by histopathology in 49 (71\%). Preoperative suspicion of adenomyosis was present in $49(20.6 \%)$ patients of all those ultimately found to have the disease.

There is a high prevalence of adenomyosis in the population studied, which indicates that the condition may have been underdiagnosed in the past, especially as it is difficult to diagnose without surgery and hysterectomy is currently the only treatment.
\end{abstract}

Adenomyosis-that is, presence of endometrial glands and stroma deep within the myometrium-is a relatively rare gynaecological diagnosis because its aetiology, diagnosis, and treatment are still not known fully. ${ }^{1}$ Only eight papers on adenomyosis appeared in Index Medicus between 1982 and 1986. More has been written on this subject recently and the number of reports on adenomyosis in Index Medicus between January 1987 and August 1989 numbered 15. Most of these reports are on newer diagnostic modalities and non-surgical intervention, ${ }^{2}$ but a few concern the prevalence of adenomyosis, of which there were five in the period 1982-89.

Little is known about the pattern of adenomyosis in Pakistani women. A review of the numbers of hysterectomies for the quality assurance committee of Aga Khan University Medical Centre indicated an unexpectedly large number of specimens with adenomyosis. We felt it worth while to report our findings in view of the limited number of reports on this subject in general and the lack of any documentation in Pakistan in particular.

\section{Methods}

The Aga Khan University Medical Centre, Karachi, is a secondary and tertiary care hospital. Patients are referred by general practitioners and gynaecologists or self referral. Most of them are local people; less than $1 \%$ of hysterectomies are performed in women of non-Pakistani origin in this community.

For the quality assurance process, the histopatholgical assessment of all hysterectomy specimens received from January 1986 to December 1989 was reviewed by one consultant pathologist. All specimens had been studied in the surgical pathology laboratory using standard histological techniques. At least three sections were taken from the uterine corpus and fundus. Adenomyosis was diagnosed if endometrial glands and stroma were found at more than one third to one fourth of the total thickness of the uterine wall away from the endometrial-myometrial junctions, as described by Hendrickson and Kempson. ${ }^{3}$ Other histopathological abnormalities were noted. Age and parity were reviewed, together with the presenting signs/ symptoms and preoperative diagnosis from the medical records of these patients. Data were analysed to study the prevalence of adenomyosis with regard to age, parity, associated pathology and confirmation of its preoperative diagnosis.

The $\chi^{2}$ test with Yates' correction was used to assess that statistical significance of differences between groups.

\section{Results}

Four hundred and nineteen hysterectomy specimens were received in the pathology laboratory. Of these, $237(56.5 \%)$ had adenomyosis according to the aforementioned criteria. The ages of patients with adenomyosis ranged from 31-74 years, a significantly higher prevalence being reported in those aged $40-59$ years $(p<0.05)$ (table 1$)$. Of these, $22(9.3 \%)$ patients were postmenopausal. The pattern of parity shown in table 2 indicates a prevalence at least as twice as high in parous compared with nulliparous women; the difference was significant $(p<0.05)$.

The analysis of other pathological entities (one or more in a single specimen) associated with adenomyosis showed uterine leiomyomas in $78(32.9 \%)$, cervicitis in $75(31.6 \%)$, and endometrial hyperplasia in $29(12.2 \%$ ) (table 3). In $54(22.7 \%)$ patients no other associated pathology was seen. A comparison of the prevalence of these features between the 
Table 1 Age distribution of patients undergoing hysterectomy

\begin{tabular}{lccc}
\hline $\begin{array}{l}\text { Age group } \\
\text { (years) }\end{array}$ & $\begin{array}{l}\text { All patients } \\
\text { No }(\%)\end{array}$ & $\begin{array}{l}\text { Patients with adenomyosis } \\
\text { No }(\%)\end{array}$ & $\begin{array}{c}\text { Prevalence of } \\
\text { adenomyosis }\end{array}$ \\
\hline$<29$ & $7(1 \cdot 7)$ & 0 & 0 \\
$30-39$ & $98(23.4)$ & $30(12 \cdot 6)$ & $30 \cdot 6$ \\
$40-49$ & $233(55 \cdot 6)$ & $164(69 \cdot 3)$ & $70 \cdot 4 \dagger$ \\
$50-59$ & $43(10 \cdot 3)$ & $32(13.5)$ & $74 \cdot 4 \dagger$ \\
$>60$ & $38(9 \cdot 0)$ & $11(4 \cdot 6)$ & 28.9 \\
All ages & $419(100)$ & $237(100)$ & 56.5 \\
\hline
\end{tabular}

«Prevalence per 100 patients undergoing hysterectomy in each age group.

$\dagger$ Prevalence in 40-59 for years age group significantly higher than other age groups $(p<0.05)$.

Table 2 Distribution of patients undergoing hysterectomy by parity

\begin{tabular}{lccc}
\hline Parity of & $\begin{array}{l}\text { All patients } \\
\text { No }(\%)\end{array}$ & $\begin{array}{l}\text { Patients with adenomyosis } \\
\text { No }(\%)\end{array}$ & $\begin{array}{l}\text { Prevalence of } \\
\text { adenomyosis }^{\star}\end{array}$ \\
\hline 0 & $18(4 \cdot 3)$ & $5(2 \cdot 1)$ & $27 \cdot 7$ \\
$1-4$ & $264(63 \cdot 0)$ & $150(63 \cdot 3)$ & $56 \cdot 8 \dagger$ \\
$>4$ & $137(32 \cdot 7)$ & $82(34 \cdot 6)$ & $59 \cdot 8 \dagger$ \\
& $419(100)$ & $237(100)$ & $56 \cdot 5$ \\
\hline
\end{tabular}

*Prevalence per 100 patients undergoing hysterectomy in each parity group.

$\dagger$ Prevalence in parous women significantly higher than nulliparous $(p<0.05)$.

group with adenomyosis and that without (table 3 ) showed a significant difference $(p<$ $0.05)$ for endometrial hyperplasia only. Its subclassification showed simple and complex hyperplasia (without atypia) in 22 and five patients, respectively, out of the 29 patients with adenomyosis, and in seven and three patients, respectively, out of the 11 without adenomyosis. Atypical hyperplastic cells were seen in only two patients in the group with, and in only one in the group without, adenomyosis.

Among the 419 patients undergoing hysterectomy, adenomyosis was stated as a single preoperative diagnosis, or in combination with other diagnoses in $69(16.4 \%)$. While 49 $(71 \%)$ turned out to have adenomyosis, the diagnosis was not verified in $20(29 \%)$ patients on histopathological examination. Put another way, out of the 237 patients ultimately found to have adenomyosis on histopathological examination, $49(20.6 \%)$ had it stated as a diagnosis before surgery.

\section{Discussion}

In developing countries an enormous number of diseases associated with poverty have undermined the clinical importance of diagnoses like adenomyosis which is difficult to confirm preoperatively, hysterectomy being

Table 3 Associated pathology in 237 patients with adenomyosis and in 182 patients without adenomyosis

\begin{tabular}{lll}
\hline Pathology & $\begin{array}{l}\text { Adenomyosis } \\
\text { No }(\%)\end{array}$ & $\begin{array}{l}\text { No adenomyosis } \\
\text { No }(\%)\end{array}$ \\
\hline Cervicitis & $75(31 \cdot 6)$ & $60(32 \cdot 9) \dagger$ \\
Endometrial hyperplasia $\dagger$ & $29(12 \cdot 2)$ & $11(6 \cdot 0) \ddagger$ \\
Uterine leiomyomas & $78(32 \cdot 9)$ & $48(26 \cdot 3) \S$ \\
Pelvic endometriosis & $11(4 \cdot 6)$ & $13(7 \cdot 1) \S$ \\
Uterovaginal prolapse & $21(8 \cdot 8)$ & $15(8 \cdot 2) \S$ \\
No other pathology & $54(22 \cdot 7)$ & $48(26 \cdot 3) \S$ \\
\hline
\end{tabular}

*One or more than one in a single specimen.

†Atypical hyperplasia seen in two patients with and in one without adenomyosis.

$\ddagger$ Prevalence significantly higher in patients with adenomyosis than in those without $(\mathrm{p}<0.05)$

§ifference insignificant $(p<0.05)$ the only diagnostic and therapeutic modality. It is no wonder that the incidence of adenomyosis is therefore not documented in Pakistan. There is a wide variation in the reported prevalence of adenomyosis as a result of the different classifications used, which are based on the invasion of myometrium by glands and stroma either in terms of proportion of uterine wall thickness or absolute measurement. ${ }^{23}$ Owing to the great variation in uterine wall thickness, we preferred to use the former and found the prevalence of $56.5 \%$ in our series to be higher than that previously reported $(10-47 \%){ }^{4}$

Most of our patients presented in the fourth and fifth decades of life; that $9 \cdot 3 \%$ were postmenopausal and $97.9 \%$ were parous is consistent with information in previously published series. ${ }^{56}$ These demographic trends in adenomyosis are similar to those of hysterectomy peak incidence in the forties and a higher prevalence in parous women. ${ }^{7}$

One patient, aged 24 , who did not undergo a hysterectomy but had a uterine septum removed surgically for treatment of recurrent abortions from a septate uterus, was found to have adenomyosis in the septum. The finding of adenomyosis at this young age is unusual but the higher number of adenomyotic foci in older patients may be related to their higher hysterectomy rates.

As noted in other reviews, ${ }^{16}$ a high percentage $(32.9 \%)$ of patients with adenomyosis had fibroids. This association, however, was equally common in the group without adenomyosis (table 3). Endometrial hyperplasia, which is more common in patients with adenomyosis, ${ }^{2}$ was present in a significantly greater proportion of our patients with adenomyosis (table 3 ). Cervicitis diagnosed by the presence of epithelial and subepithelial lymphocytic infiltrate was the other common associated finding seen in $31.6 \%$ of patients with adenomyosis, which has not been previously documented in association with adenomyosis. Cervicitis is extremely common in parous women ${ }^{8}$ and is perhaps unrelated aetiologically to adenomyosis as it was equally common in patients without adenomyosis in our series (table 3 ).

Endometriosis was present in only $4.6 \%$ of patients with adenomyosis compared with the $7 \cdot 1 \%$ prevalence in those without, but this difference was not significant $(p>0.05)$. It has been suggested that adenomyosis is a distinct disorder separate from endometriosis. $^{2}$

Preoperative suspicion of adenomyosis, based on menorrhagia, dysmenorrhoea, suitable age and parity, enlarged tender uterus and suspicion on ultrasonographic examination, was stated in $16.4 \%$ of all patients undergoing hysterectomy. The preoperative diagnosis in $20.6 \%$ of patients ultimately found to have adenomyosis in our study is as high as that of Western reports. ${ }^{69}$ The histopathological verification of the preoperative diagnosis of adenomyosis in $71 \%$ of patients in our series contrasts with the $48 \%$ verification rate reported by Lee et al. ${ }^{7}$ 
This could be explained by the high prevalence of adenomyosis in our population, which makes the probability of confirmation of its diagnosis much higher than in the West where its prevalence is much lower.

Hysterectomy continues to remain the single most important diagnostic and therapeutic procedure for adenomyosis, making it a retrospective diagnosis. With recent advances in imaging modalities and serum markers for diagnosing adenomyosis, ${ }^{2}$ it may be possible to diagnose confidently adenomyosis preoperatively. The demonstration of androgen receptors in adenomyotic foci has raised the possibility of non-surgical intervention. $^{2}$ These findings have renewed interest in adenomyosis as a clinical entity which may soon be diagnosed non-surgically and treated medically avoiding hysterectomies in a number of patients and thereby avoiding the risks of morbidity, mortality, psychological disturbances and the attendant financial pressures. This is important in a develop- ing country with limited resources such as Pakistan, where the prevalence of adenomyosis seems to be high and women are generally reluctant to have a hysterectomy.

1 Owolabi TO, Strickler RC. Adenomyosis: A neglected diagnosis. Obstet Gynaecol 1977;50:424-7.

2 Azziz R. Adenomyosis: Current perspectives. Obstet Gynecol Clin North Am 1989;16:221-35.

3 Hendrickson MR, Kempson RL. Non-neoplastic conditions of the myometrium and uterine serosa. In: Fox $\mathrm{H}$, ed. Obstetrical and gynaecological pathology. Vol 1. 3rd edn. London: Churchill Livingstone, 1987:405-7.

4 Entman SS. Uterine leiomyoma and adenomyosis. In: Jones HW, Wentz AC, Burnett LS, eds. Novack's textbook of gynaecology. 11th edn. Baltimore: Williams and Wilkins, gynaecology.

5 Bird CC, McElin TW, Manalo-Estrella P. The elusive adenomyosis of the uterus. Am J Obstet Gynecol 1972; 112:583-93.

6 Lewinski H, Emge LA. The elusive adenomyosis of the uterus. Am J Obstet Gynecol 1962;83:1541-63.

7 Lee NC, Dicker RC, Rubin GL, et al. Confirmation of the preoperative diagnoses for hysterectomy. Am J Obstet Gynecol 1984;150:283-7.

8 Lowe D, Slavin G. Non-neoplastic conditions of cervix. In: Fox H, ed. Obstetrical and gynaecological pathology, Vol 1.

9 Molitor JJ. Adenomyosis: A clinical and pathological appraisal. Am J Obstet Gynecol 1971;110:275-84. 